


\title{
Centimeter
}

$\begin{array}{llllllllllllllll}1 & 2 & 3 & 4 & 5 & 6 & 7 & 8 & 9 & 10 & 11 & 12 & 13 & 14 & 15 & \mathrm{~mm}\end{array}$ $\mid$ Inches
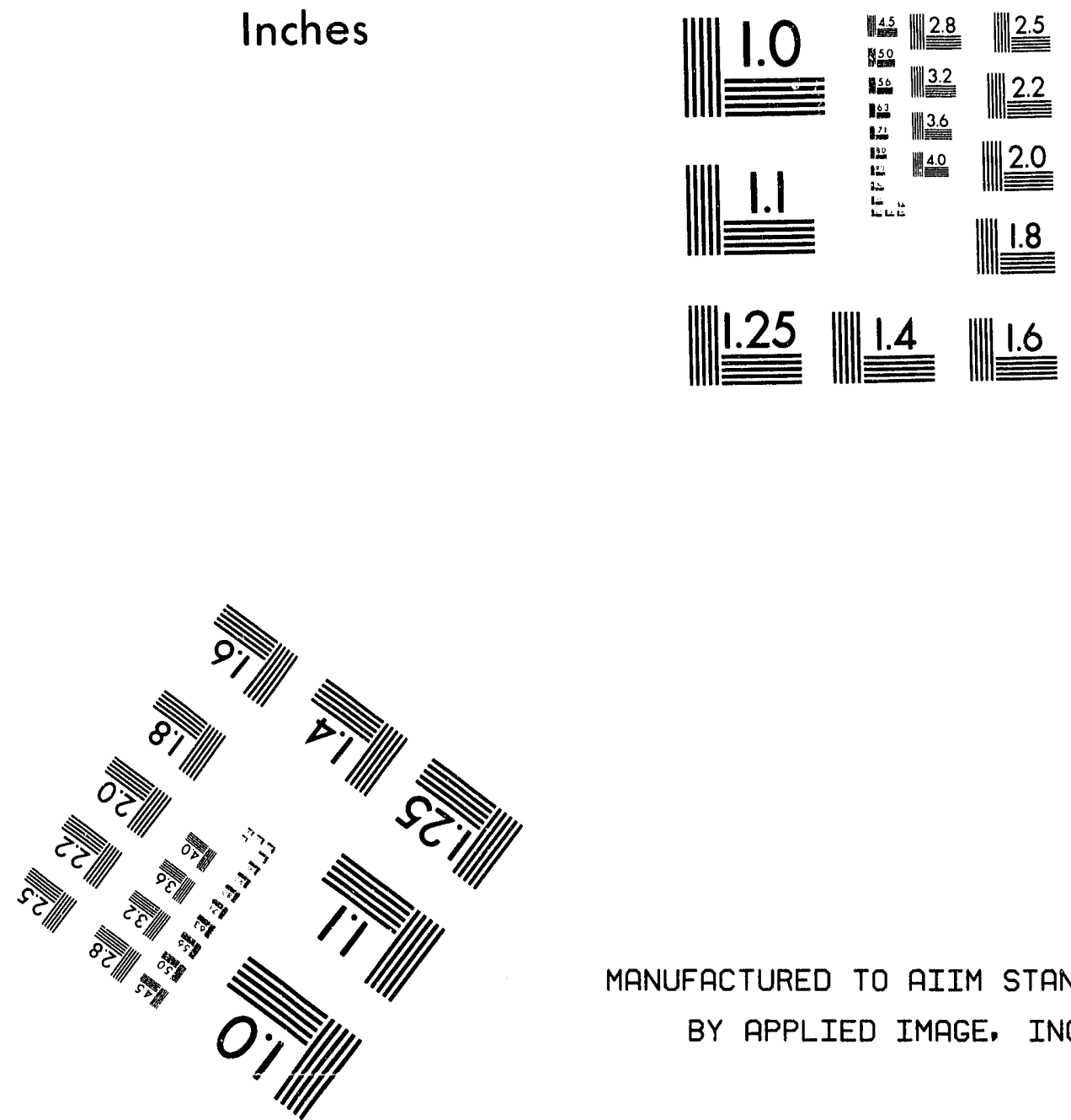

MANUFACTURED TO AIIM STANDARDS

BY APPLIED IMAGE, INC.

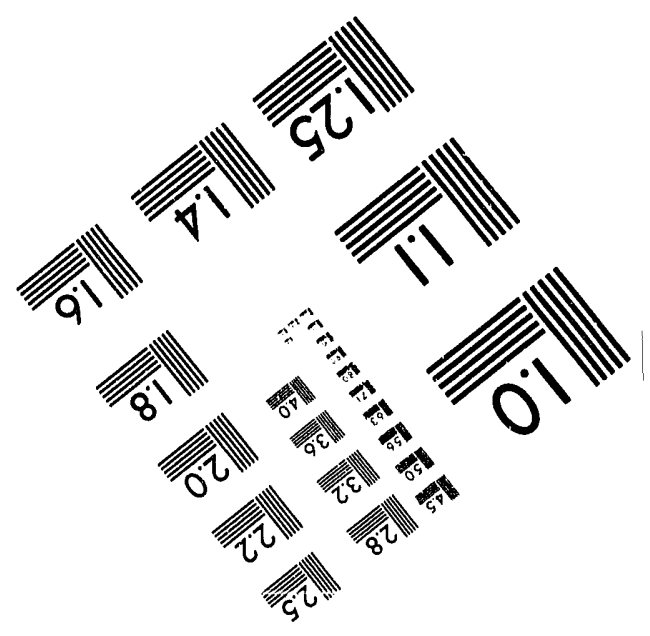



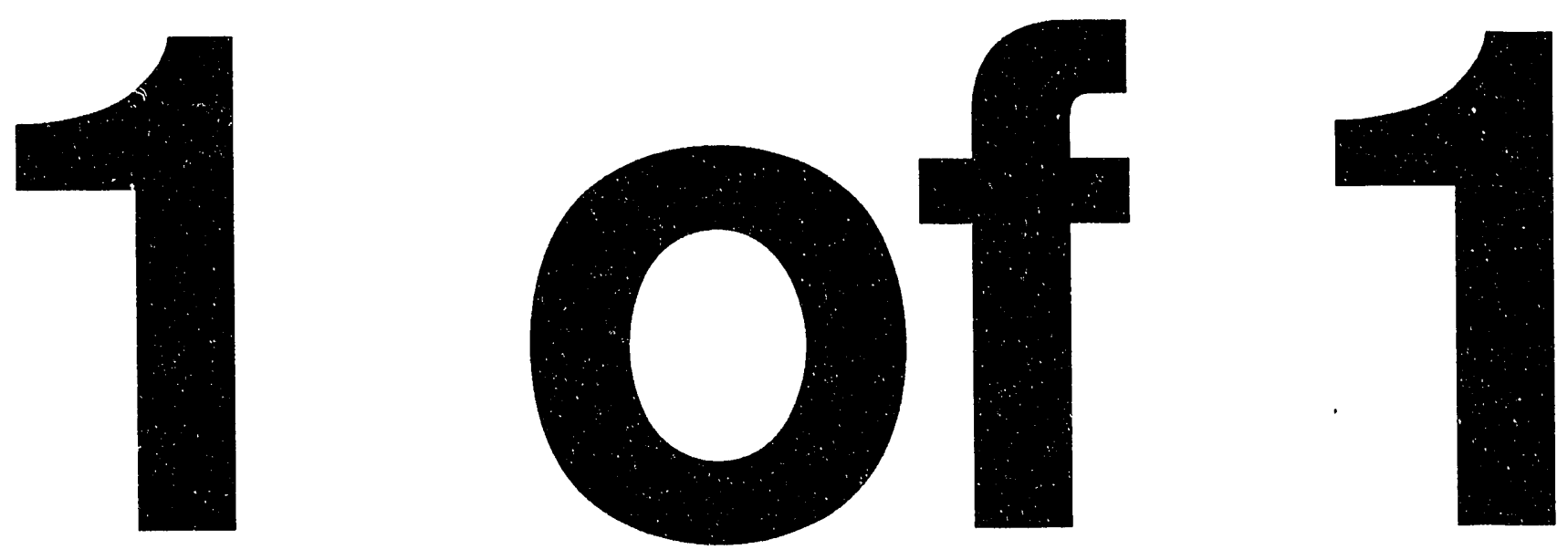


\title{
Conf-921101--123 \\ 1.20 .1
}

Note: This is a preprint of a paper being submitted for publication. Coyguts of this paper should not be quoted nor referred to without permission of the author(s). 16703

$$
03 \%
$$

\section{FAST-ICCD PHOTOGRAPHY AND GATED PHOTON COUNTING \\ MEASUREMENTS OF BLACKBODY EMISSION \\ FROM PARTICULATES GENERATED IN THE KrF-LASER ABLATION OF BN AND YBCO}

DAVID B. GEOHEGAN

Submitted to the 1992 Fall Materials Research Society Meeting,

Symposium I, Laser Ablation in Materials Processing: Fundamentals and Applications, Boston, MA

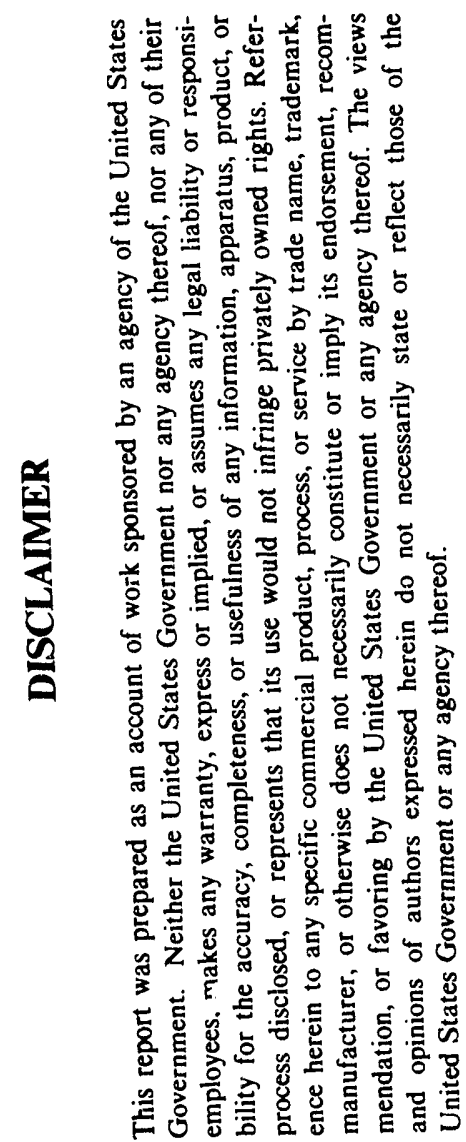

November 1992

\begin{abstract}
"The submitted manuscript has been
authored by a contractor of the U.S.

Government under contract No. DE-

AC05-84OR21400. Accordingly, the U.S.

Government retains a nonexclusive,

royalty-free license to publish or

reproduce the published form of this

contribution, or allow others to do so, for

U.S. Government purposes."
\end{abstract}

Solid State Division

Oak Ridge National Laboratory

P. O. Box 2008

Oak Ridge, Tennessee 37831-6056

managed by

MARTIN MARIETTA ENERGY SYSTEMS, INC. for the

U.S. DEPARTMENT OF ENERGY

under contract DE-AC05-84OR21400

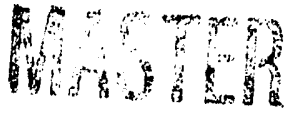




\title{
FAST-ICCD PHOTOGRAPHY AND GATED PHOTON COUNTING MEASUREMENTS OF BLACKBODY EMISSION FROM PARTICULATES GENERATED IN THE KRF-LASER ABLATION OF BN AND YBCO
}

\author{
DAVID B. GEOHEGAN
}

Solid State Division. Oak Ridge National Laboratory, Oak Ridge, TN 37831-6056

\begin{abstract}
Fast intensified CCD photography and gated photon counting following $\mathrm{KrF}$-laser irradiation of $\mathrm{YBCO}$ and $\mathrm{BN}$ targets reveals the first observations of very weak emission from slow-moving ejecta up to $2 \mathrm{~cm}$ from the target and times extending to $\approx 1.5 \mathrm{~ms}$. Time-of-flight velocities inferred from the emission measurements indicate velocities $\left(\mathrm{v} \sim(0.45-1.2) \times 10^{4} \mathrm{~cm} \mathrm{~s}^{-1}\right)$ comparable to those measured for the large particles which often accompany the pulsed laser deposition process. Gated photon counting is employed to obtain temporally resolved spectra of this weak emission. The spectral shape is characteristic of blackbody emission, which shifts to lon'er wavelengths as the particles cool during flight in vacuum. Estimates of the temperature of the particles are made based on the emissivity of a perfect blackbody and range from $2200 \mathrm{~K}$ to $3200 \mathrm{~K}$ for both $\mathrm{BN}$ and $\mathrm{YBCO}$ when irradiated at $\Phi_{248}=3.5 \mathrm{~J} \mathrm{~cm}^{-2}$ and $1.5 \mathrm{~J} \mathrm{~cm}^{-2}$, respectively. The temperature decrease of the particles in vacuum is compared to a radiative cooling model which gives estimates of the initial surface temperature and radii of the particles.
\end{abstract}

\section{INTRODUCTION}

The laser ablation process is known to produce, in addition to monatomic and diatomic neutrals and ions, large clusters and macroscopic particles. For YBCO films, the particles usually appear spherical with diameters ranging up to $10 \mu \mathrm{m}$, and are readily apparent in films deposited at low temperatures. The fraction of the mass transported to the growing film by clusters and large particulates is not known, however imp:oved superconducting thin film properties have been obtained by separating the flux of particles from the plasma plume ${ }^{1,2}$ or by vaporizing the particles using a second laser. ${ }^{3}$

Velocity filter analysis ${ }^{4}$ and laser beam deflection measurements ${ }^{1}$ have determined that the macroscopic particles travel at velocities peaked $\sim 1 \times 10^{4} \mathrm{~cm} \mathrm{~s}^{-1}$, or about 100 times slower than the velocity of the luminous plasma plume.

Several theories exist regarding the particulate ejection cause and dynamics, such as subsurface heating and eruption or expulsion by shock waves in the target. $5-7$ Few in situ diagnostics have been reported to investigate particie properties such as temperature, ejection time and size directly. 1,8

Recent imaging experiments of the laser ablation of YBCO have not observed particle ejection at very late times. ${ }^{9-14}$ ICCD images of the expansion of YBCO plasmas from the target showed a separation of the bright plasma and an easily observable glow near the target surface which lasted for $\sim 2 \mu \mathrm{s} .{ }^{9}$ Optical emission spectroscopy confirmed this emission to be from atomic and molecular species. ${ }^{15}$ Further investigation of this region using longer exposures, however, revealed that emission from the near-target region never totally extinguished. but continued to much longer times. In this paper, more sensitive measurements reveal blackbody emission from particulates generated from the $\mathrm{KrF}$ laser ablation of $\mathrm{YBCO}$ and $\mathrm{BN}$ in vacuum. ${ }^{16}$

\section{EXPERIMENTAL}

The experimental apparatus for gated-ICCD photography and optical emission spectroscopy (OES) has been previously described. ${ }^{9}, 15$ The intensified, CCD-array camera system (Princeton Instruments ICCD-576G/RB with $105 \mathrm{~mm}$ Nikkor-UV lens) was utilized without optical filters and was responsive over a $200-820 \mathrm{~nm}$ wavelength range

Measurable emission spectra could be obtained by photon counting using the direct output of a $\mathrm{t} / 9.4,1.33 \mathrm{~m}$ spectrometer outfitted with a 1800 lines $/ \mathrm{mm}$, UV holographic grating and Hamamatsu R955 photomultiplier tube. The very weak emission was easily observable as 
individual photon spikes at very late times in the $d=0-2 \mathrm{~cm}$ region following the strong laser plasma signal typically monitored by OES.

Optical emission spectra were obtained by photon counting with an $\mathrm{t} / 5.3 .0 .3$-meter spectrometer (Acton VM-503, grating: 6()$)$ lines $/ \mathrm{mm}$. $750 \mathrm{~nm}$ blaze) outtitted with a Hamamatsu R943-(02 photomultiplier. The photomultiplier was gated "off" during the first few microseconds using a gated socket and the remaining low-level signals were amplified by a tast preamplifier and then discriminated and counted by a gated photon counter (Staniord Research SR400).

The known output from a calibrated spectral source (NBS-calibrated $200 \mathrm{~W}$ quartz-halogentungsten $\operatorname{lamf}$ ) was used to determine the combined spectral efficiency of the spectrometer, photomultiplier and optics. The raw spectra were then corrected by this response curve.

\section{RESULTS}

A series of photographs is given in Figure 1 which illustrates the appearance of glowing particulates following the expansion of the plasma plume caused by $6.6 \mathrm{~J} \mathrm{~cm}^{-2} \mathrm{KrF}$ laser ablation of $\mathrm{YBCO}$ in vacuum.

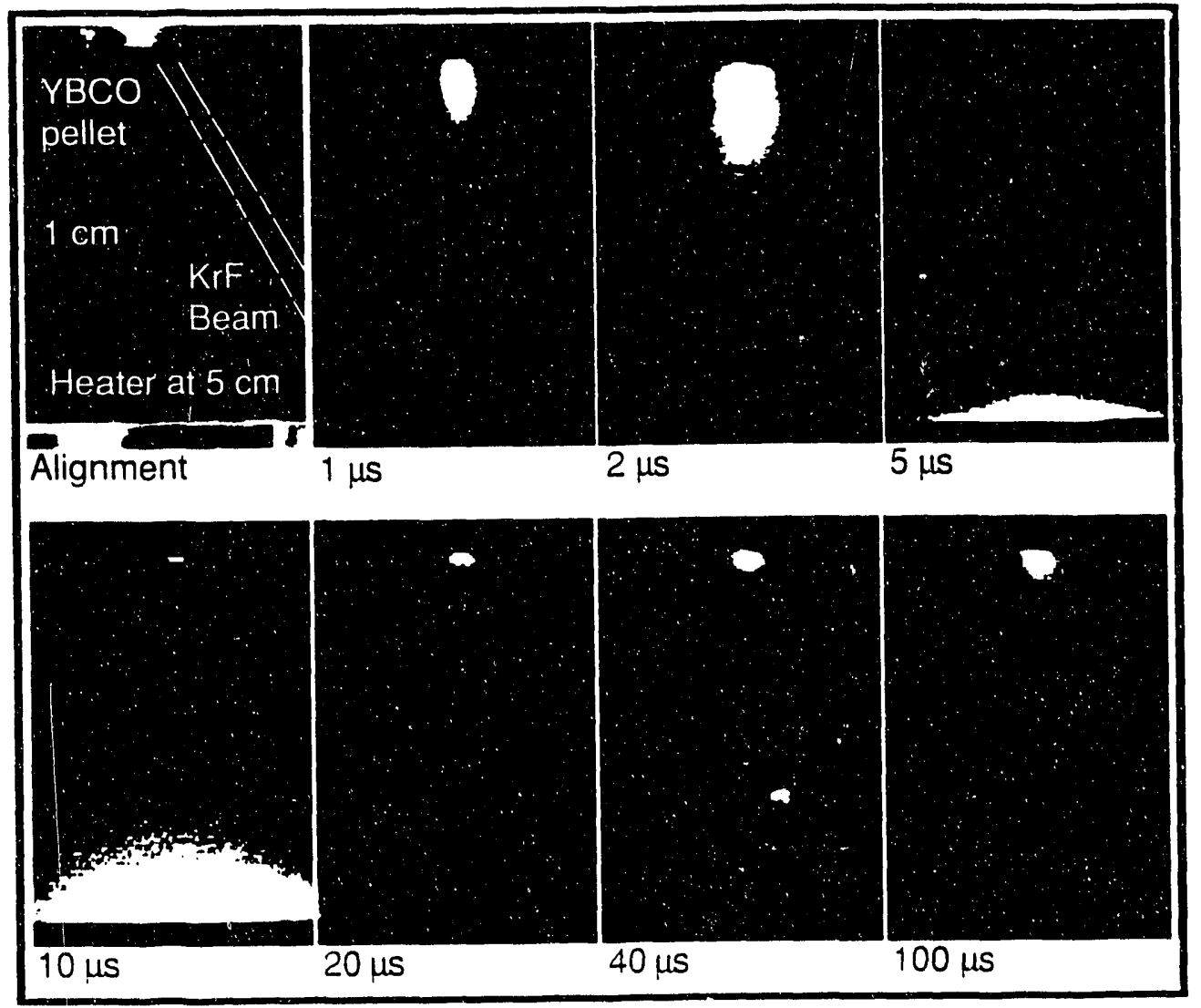

Figure 1: Photographs tracing the appearance of glowing particulates following the expansion of the plasma plume caused by $6.6 \mathrm{~J} \mathrm{~cm}^{-2} \mathrm{KrF}$ laser ablation of $\mathrm{YBCO}$ in vacuum $\left(1 \times 10^{-6}\right.$ Torr). Exposures (lens $\mathrm{t} / \mathrm{stops}$. MCP gate widths) are as follows: $1 \mu \mathrm{s}$ [t/32, 20ns]; $2 \mu \mathrm{s}[\mathrm{f} / 32.20 \mathrm{~ns}] ; 5 \mu \mathrm{s}[\mathrm{f} / 16.2() \mathrm{ns}\} ; 10 \mu \mathrm{s}[\mathrm{f} / 11,200 \mathrm{~ns}] ; 20 \mu \mathrm{s}[\mathrm{f} / 4.200 \mathrm{~ns}\} ; 40 \mu \mathrm{s}[\mathrm{f} / 8$. $1 \mu \mathrm{s}] ; \quad l(H) \mu \mathrm{s}[\mathrm{f} / 4,2.2 \mu \mathrm{s}]$.

of YBCO in vacuum. Each photograph captures a different single shot of the laser and different exposure (see caption). From $U \leq \mathrm{t} \leq 2.5 \mu \mathrm{s}$, the leading edge of the emission trom the plume travels at $2.0 \mathrm{~cm} \mathrm{\mu s}^{-1}$ from the pellet toward a cold stantless steel heater at $d=5 \mathrm{~cm}$. After the 
initial collision of the plume with the heater surface. a rebound expansion front can be seen as the brightest emission at $t=5 \mu \mathrm{s}$. The intensity of the portion of the plume which has not yet collided with the heater or the rebounded material has decreased dramatically at $t=5 \mu \mathrm{s}$. At this time. a weak glow in front of the original irradiated area can just be discerned.

At $t=10 \mu \mathrm{s}$ in Fig. 1, the glow intensity near the pellet has grown considerably in relative comparison to that of the rebounded plume. At $t=20 \mu \mathrm{s}$ and longer times the emission near the pellet suriace dominates and expands in spatial extent in an apparent secondary ejection. However, inspection of the digitized images using image processing software shows that the glow extending from the pellet suriace was present at early times, although it did not extend far nor did it dominate in intensity.

Figure 2 presents a closer view of the emission near the $Y B C O$ pellet surface with $300 \times 300$ pixel resolution over a $1.85 \mathrm{~cm} \times 1.85 \mathrm{~cm}$ imaged region. At $\mathrm{t}=5 \mu \mathrm{s}$, the plasma has already moved out of the trame and the particulates are barely extenuing $1 \mathrm{~mm}$ from the pellet surface. The fastest observed radiating particles moved $\sim 1.5 \mathrm{~cm}$ in $50 \mu \mathrm{s}\left(0.03 \mathrm{~cm} \mathrm{\mu s}^{-1}\right)$, while the

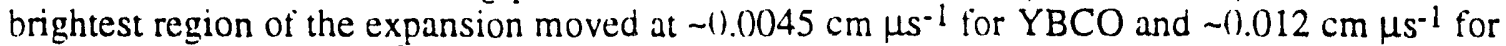
BN at $\Phi_{248}=1.5 \mathrm{~J} \mathrm{~cm}^{-2}$. The radiating particles appear to expand in a much less forwarddirected pattern than the initial plasma plume. The individual pixels observed in the photographs are likely the result of individual photons which were detected and subsequently amplified by the MCP intensifier assembly. Detectable emission exists in this region to $\sim 3 \mathrm{~ms}$ where no pixels record counts.

In order to determine the emission spectra, gated photon counting was pertormed by imaging the $d=1-10 \mathrm{~mm}$ spatial region through the opened slits of the spectrometer. The spectrometer was scanned in $5 \mathrm{~nm}$ intervals and photons were counted and averaged over at least 20 shots per wavelength.

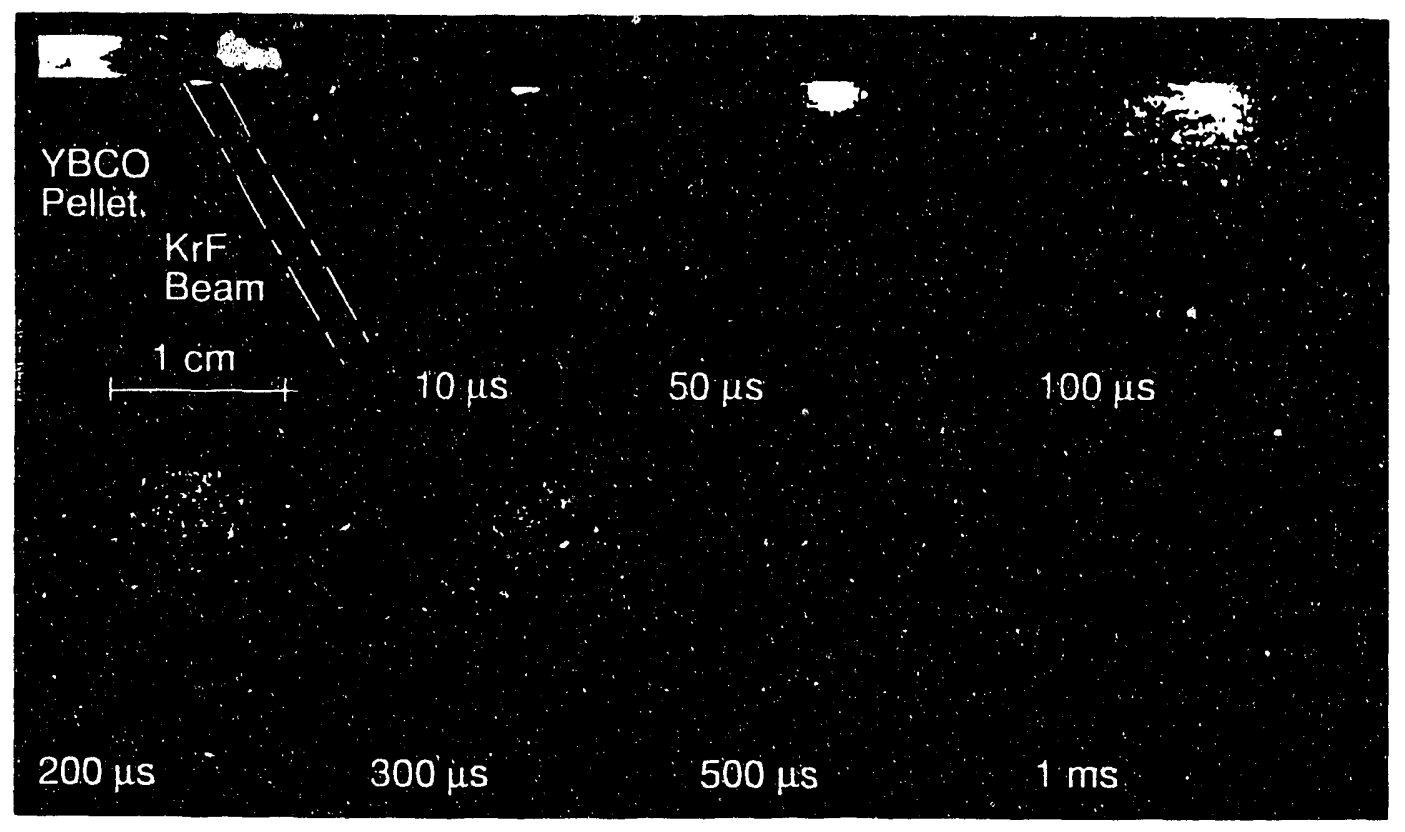

Figure 2: Photographs of glowing particulates in the $d=0-1.5 \mathrm{~cm}$ region following $1.0 \mathrm{~J}$ $\mathrm{cm}^{-2} \mathrm{KrF}$ laser irradiation of YBCO in vacuum. Time delays and exposures (lens $\mathrm{f} \#$, MCP gaie width) are as tollows: $10 \mu \mathrm{s}(\mathrm{t} / 5.6,200 \mathrm{~ns}), 5() \mu \mathrm{s}(\mathrm{f} / 4,2 \mu \mathrm{s}), 100 \mu \mathrm{s}(\mathrm{f} / 4.2 \mu \mathrm{s}), 200 \mu \mathrm{s}(\mathrm{f} / 4,2$ $\mu \mathrm{s}), 300 \mu \mathrm{s}(\mathrm{f} / 4,2 \mu \mathrm{s}), 4(00 \mu \mathrm{s}(\mathfrak{i} / 4,2 \mu \mathrm{s}), 500 \mu \mathrm{s}(\mathfrak{i} / 4,2 \mu \mathrm{s}), 1 \mathrm{~ms}(\mathfrak{E} / 4,2 \mu \mathrm{s})$.

Broad emission spectra were obtained at late times for both YBCO and BN. starting at $\sim 300$ $\mathrm{nm}$ and rising into the near-IR. Figure $3(\mathrm{a})$ shows the emission spectrum measured $60-80 \mu \mathrm{s}$ following YBCO ablation. The spectral shapes were suggestive of blackbody spectra. and curve lits to Planck's radiation law showed good agreement (see solid curve in Fig. 3(a)). 

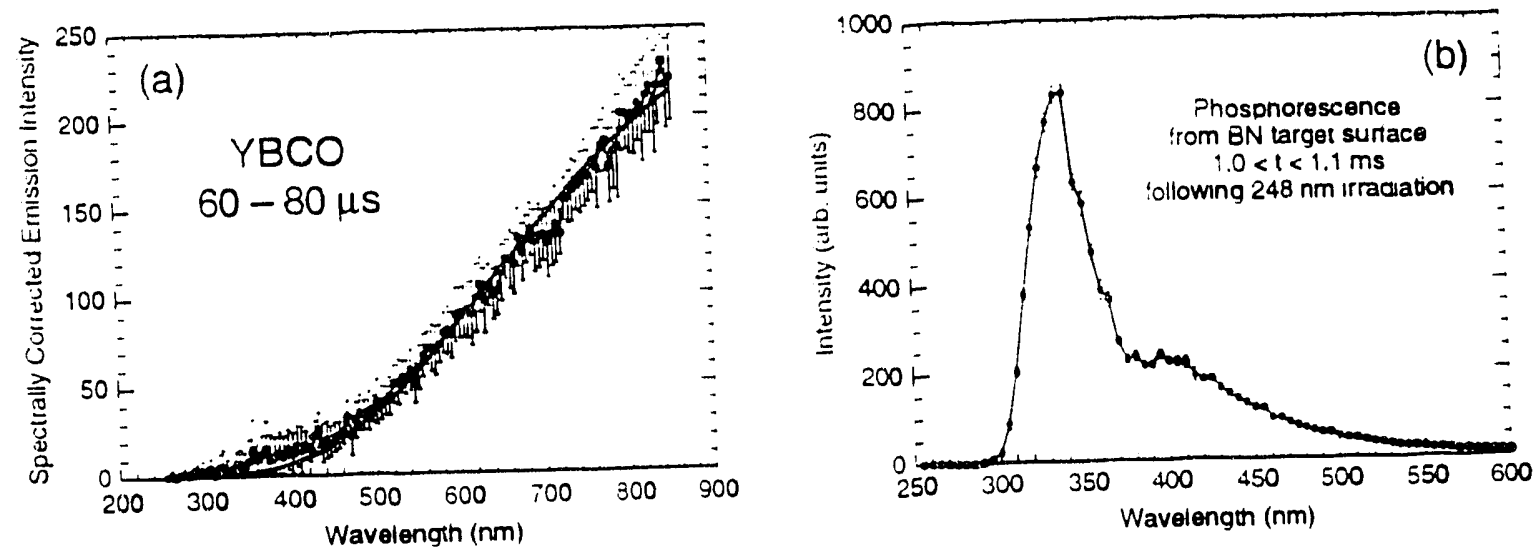

Figure 3: (a) Emission spectrum from particulates within $d=1-10 \mathrm{~mm}$ of a YBCO pellet, $60-80 \mu \mathrm{s}$ following $1.5 \mathrm{~J} \mathrm{~cm}-2 \mathrm{KrF}$ laser irradiation of $\mathrm{YBCO}$ in vacuum. The smooth curve is
a blackbody spectrum $\rho(\lambda)=\left(8 \pi \mathrm{hc} \lambda^{-5}\right)\left(\mathrm{e}^{\mathrm{he} / \mathrm{\lambda kT}}-1\right)^{-1}$ fit to the data corresponding to a perfect blackbody temperature of $2670 \mathrm{~K}$. The spectrum is corrected for the spectral response of the detection equipment (see text). Error bars represent statistical uncertainty (one standard deviation) in photons counted within each $5 \mathrm{~nm}$ step. (b) Phosphorescence spectrum from the surface of the BN pellet $1.0-1.1 \mathrm{~ms}$ iollowing $0.09 \mathrm{~J} \mathrm{~cm}^{-2} 248 \mathrm{~nm}$ irradiation in vacuum.

Other sources for the emission include luminescence from laser ablated particles. However, the time dependence and spectral shape of measured target luminescences did not correspond with the measured blackbody spectra. The BN targets utilized for these measurements displayed a blue phosphorescence easily visible to the naked eye for several seconds following the laser pulse. This phosphorescence spectrum was measured by turning the pellet and imaging the irradiated pellet zone through the spectrometer. Figure 3(b) gives the phosphorescence spectrum of the BN target surface measured 1.0-1.1 ms following $248 \mathrm{~nm}$ laser irradiation at a reduced energy density of $0.09 \mathrm{~J} \mathrm{~cm}^{-2}$. This spectrum agrees with previously published luminescence spectra. ${ }^{17}$ but does not agree with the blackbody emission measured here at any time delay. A much weaker luminescence band was measured for YBCO between $340 \mathrm{~nm}$ and $480 \mathrm{~nm}$ which does not agree with the blue photoluminescence reported following $308 \mathrm{~nm}$ excitation. ${ }^{18}$

Photon counting in the $d=1-10 \mathrm{~mm}$ spatial region at increasingly longer delays yielded emission spectra which were fit well by Planck's radiation law but indicated peaks further in the infrared. This would be expected due to cooling of the particles. As a model for the results, consider a small spherical solid particle, radius $r$, ejected from the target at temperature $T_{0}$ and its subsequent cooling by radiation as a perfect blackbody. At sufficiently high temperatures, all simple solids have the same molar specific heat given by the law of Dulong and Petit, $C_{v}=3 R=$ $25 \mathrm{~J} \mathrm{~mol}^{-1} \mathrm{deg}^{-1}$. The internal energy of the particle, radius $r$, molar density $\rho$, is

$$
E_{\text {int }}=4 / 3 \pi r^{3} \rho C_{v} T \text {. }
$$

The particle will emit radiation in accordance with the Stefan-Boltzmann Law where the total rate of energy loss per unit area of the particle is

$$
\mathrm{P}_{\mathrm{r}}=\mathrm{e} \sigma_{\mathrm{S}} \mathrm{T}^{4} \quad\left(\sigma_{\mathrm{S}}=5.67 \times 10^{-8} \mathrm{Wm}^{-2} \mathrm{deg}^{-4}\right) .
$$

The emissivity $\mathrm{e}(\lambda) \leq 1$ will be taken $\mathrm{e}=1$ for a perfect blackbody. An $\mathrm{r}=0.5 \mu \mathrm{m} \mathrm{BN}$ particle at $3000 \mathrm{~K}$, for example, will have $E_{\text {int }}=5 \times 10^{-10} \mathrm{~J}$ of energy and radiate $14 \mu \mathrm{W}$ in a blackbody spectrum which peaks at wavelength $\left(\lambda_{p}=0.97 \mu \mathrm{m}\right)$ given by Wien's displacement law. 
The cooling of the particles by blackbody radiation results in a loss of internal energy given by Eqs. (1) and (2)

$$
\frac{d E_{\text {int }}}{d t}=t / 3 \pi r^{3} \rho C_{v} \frac{d T}{d t}=-e \sigma_{s} T^{4} \cdot\left(4 \pi r^{2}\right)
$$

which can be integrated to give a radiative cooling time dependence

$$
T=\left[\frac{1}{T_{0}{ }^{3}}+\frac{3 e \sigma_{s}}{\operatorname{Rrp}} \quad 1\right]^{-1 / 3}
$$

The blackbody temperatures obtained at different time delays following laser ablation of $\mathrm{BN}$ and YBCO are plotted in Fig. 4 along with fits to the data of the form of Eq.(4). The fits to the data of Fig. 4 return $T_{0}$ and $r$, the initial temperature and radius of the particles. For BN (with $\rho=0.091$ mole $\mathrm{cm}^{-3}$ ), $\mathrm{T}_{\mathrm{O}}=3235 \mathrm{~K}$ and $\mathrm{r}=5.3 \mu \mathrm{m}$ while for $\mathrm{YBCO}$ (with $\rho=9.60 \times$ $10^{-3}$ mole $\left.\mathrm{cm}^{-3}\right), \mathrm{T}_{0}=2900 \mathrm{~K}$ and $\mathrm{r}=13.0 \mu \mathrm{m}$.
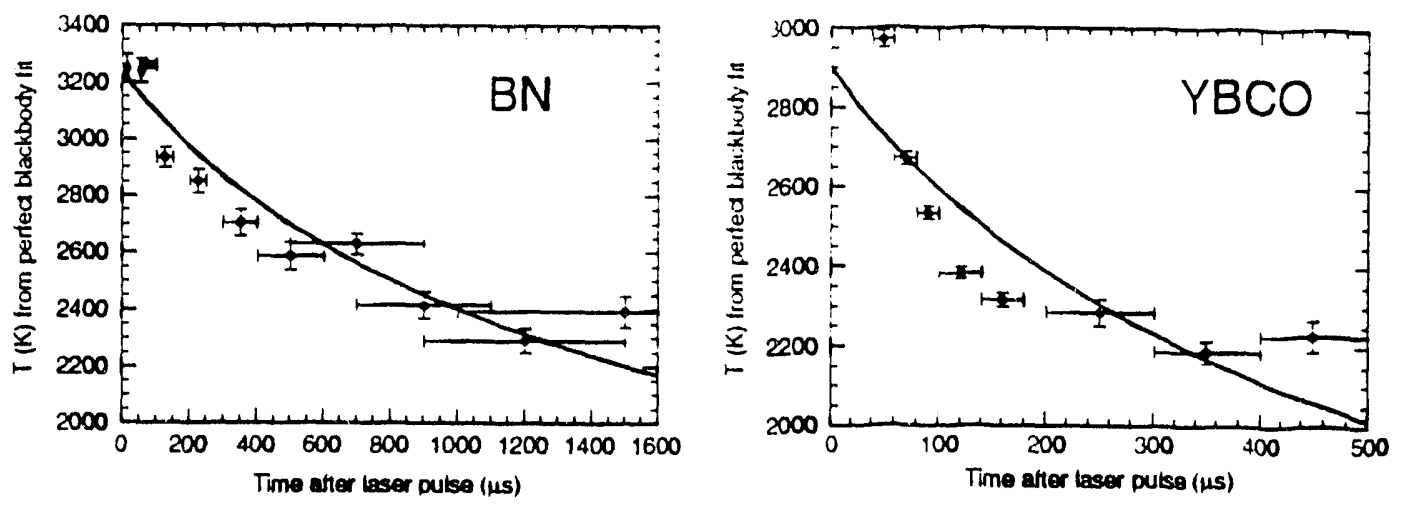

Figure 4: Particulate temperatures implied from perfect blackbody curve fits to emission spectra such as Fig. 3(a) for (a) BN and (b) YBCO at $3.5 \mathrm{~J} \mathrm{~cm}^{-2}$ and $1.5 \mathrm{~J} \mathrm{~cm}^{-2}$, respectively. Error bars indicate the temporal gate used for photon counting and the statistical uncertainty $(1 \sigma)$ in the temperatures given by the curve fits. The solid curves are fits to the data using the radiative cooling model (Eq. 4).

\section{CONCLUSIONS}

Fast ICCD photography has been used to image the emission from luminous material at very long times in the ablation of $\mathrm{YBCO}$ and $\mathrm{BN}$. The emission is also readily measurable by photon counting with optical emission spectroscopy equipment. The emission is attributed to blackbody

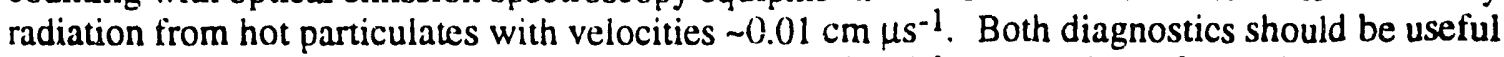
to identify and estimate the temperature for particles ablated from a variety of materials.

Initial $(t=0)$ particle temperatures from $B N$ of $T_{0} \sim 3200 \mathrm{~K}$ are implied by the blackbody emission curve tits and the radiative cooling model. This temperature is just above the $-3000 \mathrm{~K}$ sublimation temperature for BN. Some $(r-1 \mu \mathrm{m})$ particles which attached to a cool Si substrate $5 \mathrm{~cm}$ away, however, were apparently molten. Initial temperatures for particles ejected from YBCO are estimated at $\geq 2970 \mathrm{~K}$. These particle temperatures far exceed the $\sim 2270 \mathrm{~K}$ evaporation temperature of $\mathrm{YBCO}^{5}$ and are consistent with models predicting superheating of targets (for YBCO. $2 \mathrm{~J} \mathrm{~cm}^{-2}, \mathrm{KrF}: \mathrm{T} \sim 5200 \mathrm{~K}$ ) ${ }^{5}$ when rapid laser heating rates exceed thermal conductivity and evaporation kinetics. 
The simple model for the cooling of the particles assumes uniform particle size and radiative cooling only. The temperature drop of the particles from BN and YBCO are both initially faster than the radiative model suggests. Evaporation of the particles would result in additional cooling. Superheated particles should be expected to vaporize in tlight at a rate determined by the velocity of their vaporizing surface. which can be estimated from the Clausius-Clapeyron equation and a knowledge of the thermal constants of the particle material. Vaporization results in additional conling while above the evaporation temperature, in agreement with the data for BN and YBCO in Fig. 4.

The particle velocities are consistent with other experimental measurements 1,4 and with an anomalous "slow" velocity component measured for ground state neutrals following YBCO ablation. 18 Vaporization of the particles and the hot surface of the target may partially explain the very slow component noted in the ground state neutral velocity distributions and the persistence in YO density near the target after hundreds of microseconds. ${ }^{17,18}$

Rohlfing measured blackbody emission spectra following Nd:YAG laser-vaporization of graphite into a pulsed jet of helium and, using a similar model and analysis, estimated the particle size at $<100 \mathrm{~nm}$ and temperatures of $2500-4(0) 0 \mathrm{~K} .8$ In this case, the diagnostic was used to monitor the gas phase clustering reactions due to background gas collisions. Rohlfing found that the curve fits were relatively insensitive to different emissivity $\mathrm{e}(\lambda)$ dependences, but very sensitive to the temperature. $T$. Clearly, extracting detailed particle size and temperature distributions will require emissivity $\mathrm{e}(\lambda, r)$ data for small particles of the material of interest.

The technical assistance of J. K. Jackson of NIST, C. Meyers and Y. Talmi of Princeton Instruments, Inc., and G. E. Jellison. Jr., M. Abraham and E. Arakawa of ORNL is gratefully acknowledged. This research is sponsored by the Division of Materials Sciences, U.S. Department of Energy under contract DE-AC05-840R21400 with Martin Marietta Energy Systems, Inc..

\section{REFERENCES}

1. K. Murakami, p.125 in Laser Ablation of Electronic Materials: Basic Mechanisms and Applications, ed. by E. Fogarassy and S. Lazare, North Holland (1992).

2. A. A. Ivanov, S. G. Galkin, A. V. Kuznetsov, A. P. Menushenkov, Physica $C$ 180, 69 (1991).

3. G. Koren. A. Gupta, R. J. Baseman. M. I. Lutwyche, and R. B. Laibowitz, Appl. Phys. Lett. 56. 2144 (1990).

4. H. Dupendant. J. P. Gavigan, D. Givord. A. I ienard, J. P. Rebouillat, and Y. Souche, Appl. Surf. Sci.. 43, 369 (1989).

5. Deepika Bhattacharya, R. K. Singh, and P. H. Holloway, J. Appl. Phys. 70, 5433 (1991).

6. R. Kelly, J. J. Cuomo, P. A. Leary, J. E. Rothenberg, B. E. Braren and C. F. Aliotta, Nucl. Instrum. Methods B 9, 329 (1985).

7. R. K. Singh, D. Bhattacharya, and J. Narayan, Appl. Phys. Lett. 57, 2022 (1990).

8. Eric A. Rohlfing, J. Chem. Phys., 89, 6103 (1988).

9. D. B. Geohegan, Appl. Phys. Lett. 60, 2732 (1992).

10. A. Gupta. B. Braren, K. G. Casey, B. W. Hussey, and Roger Kelly, Appl. Phys. Lett. 59, 1302 (1991)

11. K. Scott. J. M. Huntiey, W. A. Phillips, John Clarke, and J. E. Field, Appl. Phys. Lett., 57, 922 (1990).

12. Daniel Fried. T. Kushida. G. P. Reck and E. W. Rothe. J. Appl. Phys. 72 1113, (1992).

13. P. E. Dyer, A. Issa. P. H. Key, Appl. Surf. Sci., 4689 (1990).

14. O. Eryu, K. Murakami, K. Masuda. A. Kasuya, and Y. Nishina, Appl. Phys. Lett. 54. 2716 (1989).

15. D. B. Geohegan. Thin Solid Films, in press.

16. D. B. Geohegan, submitted to Applied Physics Letters,

V. A. Krasnoperov, N. V. Vekshina. M. B. Khusidman. and V. S. Neshpor, Zhurnal Prikladnoi Spektroskopii 11(2) 299 (1969). Translated in J. Appl. Spectrosc. p. 931. August 1969.

18. Wang Y., Zhang X., Zhou Y.. and Wang J.. J. Lumin. 45, 165 (1990).

19. D. B. Geohegan and D. N. Mashburn, Appl. Phvs. Lett. 55. 2345 (1989). 

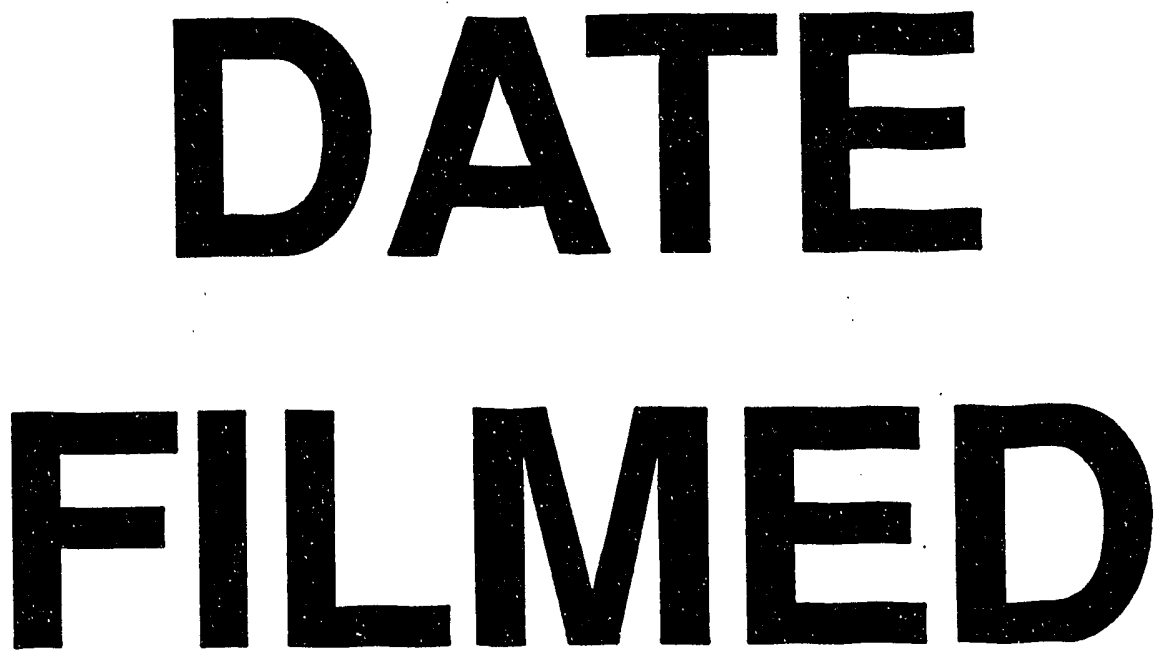

$8 / 17 / 93$

